\title{
Will helminth co-infection modulate COVID-19 severity in endemic regions?
}

\author{
Richard S. Bradbury ${ }^{1 凶}{ }^{凶}$, David Piedrafita ${ }^{1}$, Andrew Greenhill ${ }^{1}$ \\ and Siddhartha Mahanty ${ }^{2}$
}

As COVID-19 spreads through the world, most cases to date are in middle- and high-income nations. The impact on resource-poor nations remains unknown. Amongst many factors likely to affect the impact of COVID-19 in these areas, co-infections need to be considered. Here, we discuss whether the immunomodulatory effects of helminth infections may affect COVID-19 severity.

More than 1 billion people worldwide are infected with helminths, with those living in resource-poor tropical areas disproportionately affected. Complex interactions between helminths and their host result in systemic effects on immunity, with a skewing towards type 2 responses and profound consequences on the host immune milieu ${ }^{1}$. Type 2 responses suppress Thelper $1\left(\mathrm{~T}_{\mathrm{H}} 1\right)$ cells and skew cytokine response profiles towards IL-4, IL-5, IL-9 and IL-13, which are produced by expanded populations of circulating $\mathrm{T}_{\mathrm{H}} 2$ cells and alternatively activated macrophages (AAMs) ${ }^{1,2}$. Amplification of regulatory $\mathrm{T}\left(\mathrm{T}_{\mathrm{reg}}\right)$ cell and regulatory $\mathrm{B}$ cell responses further inhibits host type 1 responses ${ }^{1}$. Helminth-secreted immunomodulatory proteins induce IL-10 production and $\mathrm{T}_{\text {reg }}$ cell development and block the release of pro-inflammatory chemokines ${ }^{3}$. Moreover, helminth-induced alterations of the gut microbiome also have systemic immunomodulatory effects ${ }^{2}$. It has been demonstrated that helminth co-infection can influence the severity of viral infection in mice. Interestingly, in the case of murid herpesvirus 4 (MuHV-4) respiratory infection, prior infection with Schistosoma mansoni reduced disease severity ${ }^{3}$. However, immune responses to pulmonary coronaviruses and MuHV-4 are different and therefore the impact of helminth co-infection may differ also.

COVID-19 is caused by the betacoronavirus SARSCoV-2. In humans and mice infected with SARS-CoV, a closely related virus to SARS-CoV-2 and the causative agent of SARS, an extended duration of disease resulted in pulmonary fibrosis accompanied by perivascular infiltration and accumulation of AAMs, which are typically associated with type 2 responses ${ }^{4}$. In mice given candidate SARS-CoV vaccines, pulmonary immunopathology was associated with eosinophil infiltration, which is also characteristic of a type 2 cellular immune response ${ }^{5}$. Patients with COVID-19 who require admission to intensive care units typically have increased plasma concentrations of IL-2, IL-6, IL-7, IL-8, IL-17, G-CSF, CXCL10, CCL2, CCL3, CCL4, TNF and IFN $\gamma$ compared with those with milder disease. Notably, unlike patients with SARS, patients with COVID-19 also have elevated levels of the type 2 cytokines IL- 4 and IL-10 (REF. ${ }^{6}$ ). The involvement of type 2 responses in the immunopathology of SARS and COVID-19 is of concern when considering potential effects of helminth co-infection. We call on the research community to investigate the influence of helminth co-infection on COVID-19 outcomes as the pandemic spreads through the helminth-endemic regions of the word. Potential negative effects may influence recommendations on deworming.

1. Harris, N. L. \& Loke, P. Recent advances in type-2-cell-mediated immunity: insights from helminth infection. Immunity 47, 1024-1036 (2017).

2. Brosschot, T. P. $\&$ Reynolds, L. A. The impact of a helminth-modified microbiome on host immunity. Mucosal Immunol. 11, 1039-1046 (2018).

3. Rolot, M. et al. Helminth-induced IL-4 expands bystander memory $\mathrm{CD}^{+} \mathrm{T}$ cells for early control of viral infection. Nat. Commun. 9, 4516 (2018).

4. Channappanavar, R. \& Perlman, S. Pathogenic human coronavirus infections: causes and consequences of cytokine storm and immunopathology. Semin. Immunopathol. 39, 529-539 (2017).

5. Tseng, C. T. et al. Immunization with SARS coronavirus vaccines leads to pulmonary immunopathology on challenge with the SARS virus. PLoS One 7, e35421 (2012).

6. Huang, C. et al. Clinical features of patients infected with 2019 novel coronavirus in Wuhan, China. Lancet 395, 497-506 (2020).

Author contributions

The authors contributed equally to all aspects of the article.

\section{Competing interests}

R.S.B. is a co-patent holder of the patent WO2019060840 - Removing interfering host nucleic acids for molecular parasite detection, issued to the Centers for Disease Control and Prevention. The other authors declare no competing interests. 